\section{The Mondee Gills Game}

\section{Sasha Gnedin}

This column is a place for those bits of contagious mathematics that travel from person to person in the community, because they are so elegant, surprising, or appealing that one has an urge to pass them on.

Contributions are most welcome.
To switch or not to switch, that is the question ...

$\triangle$ game played in Mondee Gills is not so familiar because of the isolation of the country and peculiarities of the dialect of the Mondees. Many people confuse the game with its rogue variant sometimes played for money with thimbleriggers on the streets of big cities. Mainland historians trace the roots to primitive contests such as coin tossing and matching pennies. However, every Mondee knows that the game is as old as their land, and the land is really old. An ancient tomb unearthed by archaeologists on the western side of Capra Heuvel, the secondhighest hill in the country, was found to contain the remains of domesticated cave goats and knucklebones, which supports the hypothesis that the basics of the game go back to farmers of the late Neolithic Revolution.

The rules of the Mondee Gills Game (MGG) were always the same, although the scenery changed with time. Nowadays the Mondee Gills Monday TV broadcasts the game as an open-air reality show in which there are three caves, one goat, and two traditional characters called Monte and Connie. The goat is hidden by Monte in one of the caves before the show starts, two other caves are left empty. The second actor, Connie, who is randomly chosen from the audience, is ignorant of what the caves conceal, and will be offered two tries to find the goat. She is asked first to choose one of three caves. Then Monte always proceeds to reveal an empty caveone of the caves not chosen by Connie - and asks whether she wants to stay with her initial choice or switch the choice to the other remaining unrevealed cave. The goat is won if the final choice is correct.

Most visitors from nearby Laieland who are new to the game, when playing in the role of Connie, are keen on making the first choice at random, but they rarely realize the true meaning of the switch offer when two unrevealed caves remain. Many of them think that once the first choice is equally likely to fall on the cave with the goat, the equality of chances still persists when one empty cave is excluded, so the odds are 1:1 whichever the action. Some of them are reluctant to switch and prefer to stick with the first chosen cave. Others, more experienced in primitive games, flip a fair coin to decide on the second choice. The newbies quickly notice that the coin-flippers win about a half of the goats in the rounds played, whereas those who stay with the initial choice score only one third.

Needless to say, native Connies are superior in the game, managing to win about two thirds of the goats in the rounds played by switching all the time. The advantage of the Mondees, whose strategy also involves choosing the first cave uniformly at random, is due to the local educators, who developed composite teaching programs. Training starts in early childhood with a chocolate goat concealed in one of 
three bags, but it is problematic at this stage, as the kids cry when they are not given the bag they initially chose or when they are not rewarded. Later on, children practice Mondee Karlo techniques using educational computers and playing the MGG for valuable coins with goat heads and tails, either against cartoon Monte applets or by distributing the roles over two groups of players. Students of secondary schools are dedicated to the history and philosophy of the MGG [17] and have theoretical lessons on the fundamentals of the Doctrine of Chances [2].

The theory of the MGG is a subject by itself, being largely focused on the problems of utility of switch and the resolution of the paradox of odds. Basic propositions are found in Books of Texts $[1,5,15,19]$. The reader is referred to the works of Magister [6, 7, 8] for the up-to-date, critical analysis of the major theoretical developments. A rich source for those who have a good command of Mondees language is Quickygnosis [21], the online forum of the Mondee Gills educators. In this essay we shall mention only some theories that will be important for seeing the place of our contribution. The names of the two principal schools of thought can be translated from Mondees as Big-Endians and LittleEndians.

The traditional school of Big-Endians, also known as simplists, originates from prophet Prosto, who taught that paradoxes should be resolved from the convenient end. Having postulated that the first choice gives the goat with probability 1/3, Big-Endians prove that the never-switching strategy wins with this very probability $1 / 3$, and the alwaysswitching strategy wins with probability $2 / 3$ - because the revealed cave is empty, the goat is found either in the cave first chosen or in another one unrevealed. From this they conclude, quite convincingly, that the odds for the second choice cannot be 1:1 with certainty, since always-switching wins with probability strictly higher than $1 / 2$. As a further justification of the advantage of the always-switching strategy, a metaphysical experiment is conducted in which Connie's personality at the moment of the first choice

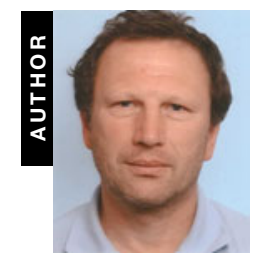

SASHA GNEDIN is (in the reverse lexicographic order) a Russian-German-DutchBritish mathematician doing research and teaching in the fields of probability, optimization, games, and combinatorics. Being an adept of Concrete Mathematics he especially likes working on the easy-to-formulate classical problems commonly viewed as "solved". Outside mathematics, he enjoys travel, tennis, and playing blues on guitar.

Department of Mathematics
Utrecht University
Post Office Box 80010
Utrecht, 3508 TA
The Netherlands
e-mail: A.V.Gnedin@uu.nl

dissociates in two, of which Jekyll-Connie sticks with the first choice, whereas Hyde-Connie switches. A newest branch of the school goes farther to assert that the odds of the second choice do not exist unless it is explicitly specified what kind of random device Monte is using when the first Connie's choice was correct and he is free to decide which of two empty caves he is going to reveal. Despite the internal split, all Big-Endians consider the paradox of odds completely resolved by these arguments, and the utility of switch justified.

Formerly, everyone accepted the simplistic views, but, a few generations in the past, the princess of Mondee Gills had bad luck in playing the game. The legend narrates that she chose cave 1, and when Monte opened cave 3 she switched to cave 2 , whereas the golden goat was hidden in cave 1 . To console the princess, the duke, a devoted friend of the ruling house, passed a law to increase the number of caves from 3 to 1000. But on the next Monday the princess lost again by switching to cave 2 after all caves numbered from 3 to 1000 had been revealed as empty. Based on conclusions of the investigation commissioned by the duke, the High Court accused Monte of fraud, and Monte, who was in fact the minister of education, was fired. Later studies showed that the minister was right when he murmured that such misfortune is not unlikely to occur to some Connies, given the fact that the MGG is played every Monday, but this theme will lead us away from our main topic... The ruler at that time, father of the princess, overruled the duke's law and issued a decree commanding all to stick with tradition by playing exclusively with 3 caves, and for the host to flip a fair coin for revealing one of two empty caves when Connie's first guess is correct. The decree called into life the Little-Endian school of conditionalistic thinking. The wisdom of the ruler enabled the scholars to define the odds for Connie's second choice with perfect mathematical rigor and eventually to justify the advantage of switching by evaluating the odds in its favor at 2:1 in the particularly important case when cave 1 was chosen by a random pick and cave 3 revealed. More advanced modern methods showed that under this mode of play, the result 2:1 also holds for other selections of two distinct cave numbers.

The schools of Big-Endians and Little-Endians are in a state of constant controversy, each accusing the other of resolving the odds paradox from the wrong end. The LittleEndians claim that their opponents do not provide enough evidence that switching is a better action for Connie after she has chosen cave 1 and cave 3 has been revealed. The differences have grown so large as to threaten the country's educational activities, leading the High Court to ban some of the most offensive scholars from Quickygnosis to cool down the mood [22].

One insightful, unorthodox offspring of the Little-Endian paradigm is the Monte Crawl game [18], in which Monte always crawls to reveal the cave with smaller number from the two caves unchosen by Connie, when her initial choice happens to be correct. Under this mode of play, Connie receives a definite signal about the location of the goat when Monte is forced to go to the cave with the larger number, because when he cannot reveal the cave with the smaller number the goat is certainly there. When it comes to Connie's 
second guess, the odds in favor of switching are no longer constant, as with the fair-coin-flipping Monte, rather they stay 1:1 if the cave with smaller number is revealed, but 1:0 if the cave with larger number is revealed. A dual regime is the Monte Haul game, in which Monte is always hauled to the cave with higher number to reveal it when Connie's initial choice happens to be correct, the odds being 1:0 and 1:1, respectively. These instances of Monte's behavior are important for seeing that the odds can be 1:1 in some situations, but under no circumstances do the odds strictly favor sticking with the initial choice, provided that the initial guess is correct with probability $1 / 3$ (and assuming that the odds for the second choice are well defined).

One subtle distinction between two major theories, which is rarely stated explicitly, is that the Big-Endianness only admits two of Connie behaviors, which may be called constant-action strategies: always switch, or always stick. The Big-Endian solution states the superiority of always-switching over always-sticking by the same initial choice of the cave. In contrast to that, the Little-Endianness admits dependence of the last Connie action on what happened so far, thus allowing for mixed-action (or history-dependent) strategies - perhaps sometimes switch and sometimes not; the task of the theory amounts then to showing that always switching is optimal within the larger class of strategies.

In this connection we should warn the reader against possible misinterpretations of the name of our principal character. The name Connie should not be associated with "contestant", rather it stems from Constance, meaning the actor winning by playing constant-action, always-switching strategies.

A major actual breakthough in the odds paradox is found in the newest writings of Magister [8], where the Little-Endian solution is derived from the Big-Endian with the aid of the principles of symmetry. The present article is devoted to another line of Magister's thought in the direction of viewing MGG as a game of strategy, that is, a situation in which two actors interact to achieve their goals within the framework specified by the rules of the game. We credit Professor [5] for the evaluation of Connie's winning probability in the worst case as being 2/3, and for identifying whcih of Monte's strategies is most unfavourable for her, namely, his rolling a symmetric three-sided die to determine the goat's hiding place. This key result and its simple proofs are ranked by Magister [9] as the Holy Grail of the MGG studies, not to say that it sets objective limits to the long-run proportion of goats won from a hostile Monte willing to keep as many goats as possible.

\section{Middle-Endianness: A Simplistic Path}

The approach we take here may be called Middle-Endianness, because it is equidistant from the two mainstreams, and it circumvents the probabilistic postulates in the problem of the utility of switching. The new doctrine is an instance of the fundamental principle of eliminating dominated strategies; in simplistic terms it can be stated as follows: No matter where Monte hides the goat and how he plays when he can choose between two caves to reveal, each Connie strategy "choose cave $Y$ and stay with it" is outperformed by a strategy "choose cave $Y^{\prime} \neq Y$, then switch". We believe that this mode of thinking has the potential to provide a convincing explanation of the advantage of the switching action to the people from Laieland, as compared with more sophisticated Big-Endian/Little-Endian and other methods based on computing probabilities. Once Laiemen adopt strategic thinking and realize that there is a two-step action, the comparison of alternatives becomes obvious and, moreover, free of any probability considerations.

To conceive the twist in the switch-versus-notswitch dilemma we stay in this introduction with the simplistic scenario, that is, ignore which particular cave is revealed by Monte in the case of an initial correct guess. Let $X$ denote the cave hiding the goat. Consider the following three strategies for Connie:

$A$ : choose cave 1 , do not switch,

$B$ : choose cave 1 , then switch,

$C$ : choose cave 2 , then switch.

Strategy $A$ wins if $X=1$, whereas strategy $B$ wins if $X \in$ $\{2,3\}$, so the strategies $A$ and $B$ cannot win simultaneously. The odds are 1:2 against $A$ if the values of $X$ are assumed to be equally likely. More generally, the extended Big-Endian theory assigns arbitrary probabilities to the values of $X$ and leads to the familiar conclusion that $B$ should be preferred to $A$ provided the probability of $X=1$ is less than the probability of $X \in\{2,3\}$. Nothing new so far, but now including $C$ into the consideration we observe that strategy $C$ wins for $X \in\{1,3\}$, so if $A$ wins then $C$ wins too, and there is a situation when $C$ wins while $A$ fails. Thus strategy $C$ is not worse than $A$, and it is strictly better if cave 3 sometimes hides the goat. This provides a general ground to avoid $A$, and for a similar reason to avoid all other strategies that do not switch in some situation.

Experimentation with strategies $A$ and $C$ exercised simultaneously is possible by complex metaphysics. Before the game starts, Connie's personality splits into Jekyll-Connie to play $A$ and Hyde-Connie to play $C$, and the dissociated players will not communicate. Monte hides the goat in one of the caves, and then Jekyll-Connie and Hyde-Connie make their first choices according to strategies $A$ and $C$, respectively. On the next move Monte reveals for Jekyll-Connie an empty cave from caves 2 and 3, and he reveals for HydeConnie an empty cave from caves 1 and 3. Based on their initial choices and their private information received from Monte, the dissociated players proceed with a final move according to their strategies. No matter which rule Monte uses to reveal the cave, Hyde-Connie will only lose when the goat is in cave 2, but in this case Jekyll-Connie will lose, too, as she does not switch to cave 2 according to strategy $A$. Following a suggestion by Magister [9], we may call this way of comparing strategies coupling by the initial position (i.e., location of goat). The coupling is analogous to simultaneously playing the same strategy in chess with, say, white pieces against two players on two boards.

We stress that, according to the Big-Endian/Little-Endian theory, discarding $A$ in favor of $B$ must be based on the probabilities of the mutually exclusive events $X=1$ and $X \in$ $\{2,3\}$. In the Middle-Endian theory, the advantage of $C$ over 
$A$ follows from the fact that the event $X=1$ is included in the event $X \in\{1,3\}$, which is much stronger as the win of $A$ implies the win of $C$.

The dominance extends our horizons in understanding the Mondee Gills Game. For instance, thinking of the superiority of $C$ over $A$, we do not need to interpret sometimes as "having positive probability". Think of a computer program that schedules in a deterministic manner the location of the goat, and suppose that on some Mondays the goat is hidden behind cave 3 . Then $C$ will have a strict advantage over $A$ because of these very Mondays. In the following discussion we shall only focus on application of the Middle-Endian paradigm to the antagonistic variant of the game $[5,7]$.

\section{The Zero-Sum Game}

We shall consider the interaction of Monte and Connie as a game of strategy, as opposed to a game against nature. Monte has freedom to choose the cave hiding the goat and to reveal one of the empty caves when he has two options. Connie chooses a cave, and when offered the second choice decides between switch and notswitch. Connie wants to win the goat. We shall no longer confine ourselves to the simplistic scenario, rather we allow arbitrary dependence of the actions on the course of the game. A fundamental model of interaction is the actors' pure competition for goats, because this instance reveals what Connie can achieve under the least favorable circumstances. A technical term for this is a zerosum game, in which at the start of the game the goat belongs to Monday TV and Monte wants to keep the goat for the program.

Now we need to make the exposition more formal, so as to avoid possible misinterpretations of Middle-Endianness. Probabilities will be introduced into the scene as mixed strategies of two active actors, Monte and Connie. Although this article is mainly self-contained, we expect that the reader is familiar with the basic concepts of strategy, minimax solution, payoff, and common knowledge. For these and the propositions used in the following text, we refer to the online Course on Games [4].

\section{Strategies and the Payoff Matrix}

To introduce notation for the possible actions of actors and to formalize the rules, it will be convenient to label the caves 1 , 2,3 in the left-to-right order. The game in extensive form has four moves:

(i) Monte chooses a cave $X$ out of 1,2,3 in which to hide the goat. The choice is kept secret.

(ii) Connie picks a cave $Y$ out of 1, 2, 3 and announces her choice. Now both actors know $Y$, and they label the caves distinct from $Y$ Left and Right in the left-to-right order.

(iii) If $Y=X$, so the choice by Connie fell on the cave with the goat, Monte chooses cave $Z$ to reveal from the Left and Right caves. In the event of the mismatch $Y \neq X$, Monte reveals the remaining cave $Z$ (distinct from $X$ and $Y$ ), which is either Left or Right depending on $X, Y$.

(iv) Connie observes the revealed cave $Z$ and makes a final decision: she can choose between Notswitch or Switch from $Y$ to another unrevealed cave (so distinct from $Y$ and $Z$ ). Connie wins if the final choice yields $X$ and loses otherwise.

Monte's action on step (iii), when he has the freedom of choosing to reveal either the Right or Left cave, may depend on Connie's initial choice $Y$. Connie's final action in (iv) depends on both $Y$ and $Z$. The rules of the game are part of the common knowledge, which means that everybody knows the rules, and knows that everybody knows, and knows that everybody knows that everybody knows, etc.

To put the game in matrix form, we label the admissible pure strategies of the actors. The pure strategies of Monte are

$$
\text { 1L, 1R, 2L, 2R, 3L, 3R. }
$$

For instance, according to strategy $2 \mathrm{~L}$ the goat is hidden in cave $X=2$, then if the outcome of (ii) is $Y=2$, Monte will reveal the Left cave (which is cave 1). Otherwise the second move of Monte is forced and the "L" part of the code $2 \mathrm{~L}$ is irrelevant, in particular if $Y=1$ he will reveal cave 3 (which happens to be the Right cave), and if $Y=3$ he will reveal cave 1 (which happens to be the Left cave).

The pure strategies of Connie are

$$
\begin{aligned}
& 1 \mathrm{SS}, 1 \mathrm{SN}, 1 \mathrm{NS}, 1 \mathrm{NN}, 2 \mathrm{SS}, 2 \mathrm{SN}, 2 \mathrm{NS}, 2 \mathrm{NN}, 3 \mathrm{SS}, \\
& 3 \mathrm{SN}, 3 \mathrm{NS}, 3 \mathrm{NN} \text {. }
\end{aligned}
$$

The digit 1,2 , or 3 is a value of $Y$, whereas SS, SN, NS, NN encode how Connie's second action depends on $Y$ and whether the Left or Right cave is revealed. For instance, 1NS means that cave $Y=1$ is initially chosen, then Connie plays Notswitch if Monte reveals the Left cave; and she plays Switch if Monte reveals the Right cave.

The game is played as if Monte and Connie have specified their two-step pure strategies before the Mondee Gills show starts. For this purpose they may ask friends for advice or employ random devices such as spinning a roulette wheel or rolling dice. After the choices are made the actors just follow their plans. The choices could be communicated to a referee who announces the then predetermined outcome of the game. For example, if Connie and Monte choose profile (2SN, 1R) the show proceeds as follows:

(i) Monte hides the goat in cave 1 .

(ii) Connie picks cave 2, thus the actors label cave 1 as Left and cave 3 as Right.

(iii) Monte observes a mismatch, hence he reveals the remaining cave 3 .

(iv) Connie observes revealed cave 3, which is Right, hence she plays Notswitch - meaning that she stays with cave 2 (and loses).

In the zero-sum game the payoff of one actor is the negative of the payoff of the other: Connie wants to win the goat while Monte aims to avoid this. With regard to the satisfaction of actors there are two distinguishable outcomes - so we agree that Connie's payoff is 1 if she wins the goat and 0 otherwise. All possible outcomes of the game are summarized in matrix $\boldsymbol{C}$ showing Connie's payoffs. The matrix has appeared in Professor's Book of Texts [5]: 


\begin{tabular}{c|cccccc} 
& $1 \mathrm{~L}$ & $1 \mathrm{R}$ & $2 \mathrm{~L}$ & $2 \mathrm{R}$ & $3 \mathrm{~L}$ & $3 \mathrm{R}$ \\
\hline 1SS & 0 & 0 & 1 & 1 & 1 & 1 \\
$1 \mathrm{SN}$ & 0 & 1 & 0 & 0 & 1 & 1 \\
1NS & 1 & 0 & 1 & 1 & 0 & 0 \\
$1 \mathrm{NN}$ & 1 & 1 & 0 & 0 & 0 & 0 \\
& & & & & & \\
2SS & 1 & 1 & 0 & 0 & 1 & 1 \\
2SN & 0 & 0 & 0 & 1 & 1 & 1 \\
2NS & 1 & 1 & 1 & 0 & 0 & 0 \\
2NN & 0 & 0 & 1 & 1 & 0 & 0 \\
& & & & & & \\
3SS & 1 & 1 & 1 & 1 & 0 & 0 \\
$3 \mathrm{SN}$ & 0 & 0 & 1 & 1 & 0 & 1 \\
3NS & 1 & 1 & 0 & 0 & 1 & 0 \\
$3 \mathrm{NN}$ & 0 & 0 & 0 & 0 & 1 & 1
\end{tabular}

The mathematical structure of the game will not be changed by certain transformations of the payoff matrix. For instance, we might replace each 0 by -1 . The latter would correspond to the variant of the game in which both Connie and Monte bring to the show one of their own goats, which are then hidden by Monte in the same cave. Both goats go into the possession of Connie, respectively Monte, depending on whether Connie finds the cave with goats or not.

A mixed strategy for Connie is a row vector $\boldsymbol{P}$ of twelve probabilities that are assigned to her pure strategies. Similarly, a mixed strategy for Monte is a row vector $\boldsymbol{Q}$ with six components. When strategy profile $(\boldsymbol{P}, \boldsymbol{Q})$ is played by the actors, the expected payoff for Connie, equal to her winning probability, is computed by matrix multiplication as $\boldsymbol{P C Q}^{T}$, where ${ }^{T}$ denotes transposition. This way of computing the winning probability presumes that the actors' choices of pure strategies are independent random variables, which may be simulated by their private randomization devices. Intuitively, the statistical independence is a way to say that cooperation of the actors in zero-sum games is impossible, so that the actors cannot agree on any other kind of joint distribution over the outcomes.

Suppose for a while that Monte is determined to play some given strategy $\boldsymbol{Q}$ and that everybody including Connie knows this. Then her optimal behavior is $\boldsymbol{P}^{\prime}$, a strategy of best response (aka Bayesian strategy) against $\boldsymbol{Q}$ to maximize $\boldsymbol{P} C \boldsymbol{Q}^{T}$ :

$$
\boldsymbol{P}^{\prime}=\boldsymbol{P}^{\prime}(\boldsymbol{Q}), \quad \boldsymbol{P}^{\prime} C \boldsymbol{Q}^{T}=\max _{\boldsymbol{P}} \boldsymbol{P} C \boldsymbol{Q}^{T} .
$$

Exchanging the roles of the actors, we suppose that Connie is determined to test a particular $\boldsymbol{P}$ and tells this to everybody, so that Monte can react with a best response $\boldsymbol{Q}^{\prime}$ :

$$
\boldsymbol{Q}^{\prime}=\boldsymbol{Q}^{\prime}(\boldsymbol{P}), \quad \boldsymbol{P} C \boldsymbol{Q}^{\prime T}=\max _{\boldsymbol{Q}} \boldsymbol{P C} \boldsymbol{Q}^{T} .
$$

A strategy profile $\left(\boldsymbol{P}^{*}, \boldsymbol{Q}^{*}\right)$ is said to be a game solution (or minimax solution) if the strategies are the best responses to each other,

$$
\boldsymbol{P}^{*} \boldsymbol{C} \boldsymbol{Q}^{* T}=\max _{\boldsymbol{P}} \boldsymbol{P} \boldsymbol{C} \boldsymbol{Q}^{* T}=\min _{\boldsymbol{Q}} \boldsymbol{P}^{*} \boldsymbol{C} \boldsymbol{Q}^{T} .
$$

The common value is the uniquely determined (so the same for all minimax profiles) value of the game, which we denote $V$. The existence of the solution is the Minimax Theorem (see [2], p. 197 for the history).

\section{Dominance}

The search for a solution is facilitated by a simple reduction process based on the Middle-Endian idea of strategic dominance. An actor's strategy $A$ is said to be weakly dominated by this actor's strategy $B$ If anything the actor can achieve using strategy $A$ can be achieved at least as well using $B$, no matter what the opponent does. That is to say, for every counterstrategy $S$ of the opponent, the outcome of the game with $B$ played against $S$ is at least as favorable as the outcome of the game with $A$ played against $S$. If $A$ and $B$ are pure strategies of Connie, the dominance simply means that if row $A$ of the payoff matrix has 1 in some column then row $B$ also has 1 in this very column.

The principle of eliminating dominated strategies takes the form of a theorem, which says that reduction of the game matrix by removal of weakly dominated pure strategies (rows or columns) does not affect the value of the game. It is a good exercise to derive the principle from the Minimax Theorem. This enables us to reduce $\boldsymbol{C}$ by noticing that $1 \mathrm{SS}$ dominates $2 \mathrm{SN}$ and $2 \mathrm{NN}$ :

\begin{tabular}{c|cccccc}
$1 \mathrm{SS}$ & 0 & 0 & 1 & 1 & 1 & 1 \\
$2 \mathrm{SN}$ & 0 & 0 & 0 & 1 & 1 & 1 \\
$2 \mathrm{NN}$ & 0 & 0 & 1 & 1 & 0 & 0
\end{tabular}

and that 3 SS dominates 2NS:

\begin{tabular}{l|llllll}
$3 \mathrm{SS}$ & 1 & 1 & 1 & 1 & 0 & 0 \\
$2 \mathrm{NS}$ & 1 & 1 & 1 & 0 & 0 & 0
\end{tabular}

Similarly, all $Y \mathrm{NS}, Y \mathrm{NN}$, and $Y S \mathrm{~N}$ strategies are dominated for $Y=1,2,3$. After row elimination, the original game matrix $C$ is reduced to a smaller matrix:

\begin{tabular}{c|cccccc} 
& $1 \mathrm{~L}$ & $1 \mathrm{R}$ & $2 \mathrm{~L}$ & $2 \mathrm{R}$ & $3 \mathrm{~L}$ & $3 \mathrm{R}$ \\
\hline $1 \mathrm{SS}$ & 0 & 0 & 1 & 1 & 1 & 1 \\
$2 \mathrm{SS}$ & 1 & 1 & 0 & 0 & 1 & 1 \\
$3 \mathrm{SS}$ & 1 & 1 & 1 & 1 & 0 & 0
\end{tabular}

Note that the strategies involving Nonswitch action are all gone!

Continuing the reduction process, we observe that columns $X \mathrm{R}$ and $X \mathrm{~L}$ of the reduced matrix are identical for $X=1,2,3$, hence, using dominance, now from the perspective of Monte, the matrix can be further reduced to the square matrix $c$ :

\begin{tabular}{c|ccc} 
& $1 \mathrm{~L}$ & $2 \mathrm{~L}$ & $3 \mathrm{~L}$ \\
\hline 1SS & 0 & 1 & 1 \\
2SS & 1 & 0 & 1 \\
3SS & 1 & 1 & 0
\end{tabular}




\section{Mismatching the Caves and the Monte Crawl Solution}

The matrix $c$ is the structure of payoffs in the Big-Endian theory, concerned with the constant-action strategies. Each actor has only three pure strategies. Monte and Connie simultaneously choose caves; if the choices mismatch Connie wins, otherwise there is no payoff.

The game has no solution in pure strategies (saddle point), thus we turn to the actors' mixed strategies $\boldsymbol{p}, \boldsymbol{q}$, which we write as vectors of size three. One may guess and then check that if Connie plays the mixed strategy with probability vector $\boldsymbol{p}^{*}=(1 / 3,1 / 3,1 / 3)$, then her probability of winning is $2 / 3$, no matter what Monte does. It is sufficient to check this for three products $\boldsymbol{p}^{*} \boldsymbol{c} \boldsymbol{q}^{T}$, where $\boldsymbol{q}$ is one of Monte's pure strategies, $(1,0,0),(0,1,0),(0,0,1)$. Similarly, if Monte plays the mixed strategy $\boldsymbol{q}^{*}=(1 / 3,1 / 3,1 / 3)$, then Connie's winning probability is $2 / 3$ no matter what she does. Connie can guarantee winning chance $2 / 3$, and Monte can guarantee that the chance is not higher, therefore $V=2 / 3$ is the minimax value of the reduced game, that is,

$$
\max _{\boldsymbol{p}} \min _{\boldsymbol{q}} \boldsymbol{p} \boldsymbol{c} \boldsymbol{q}^{T}=\min _{\boldsymbol{q}} \max _{\boldsymbol{p}} \boldsymbol{p} \boldsymbol{c} \boldsymbol{q}^{T}=\boldsymbol{p}^{*} \boldsymbol{c} \boldsymbol{q}^{* T}=2 / 3 .
$$

Instead of guessing the minimax probability vectors $\boldsymbol{p}^{*}$ and $\boldsymbol{q}^{*}$ one could use various computational techniques found in Chapter 3 of Course on Games [4]. Here, we mention one insightful transformation that should convince the reader that the solution is correct. Subtracting from $c$ the constant matrix with all entries equal to 1 reduces to the game with diagonal matrix

\begin{tabular}{c|ccc} 
& $1 \mathrm{~L}$ & $2 \mathrm{~L}$ & $3 \mathrm{~L}$ \\
\hline $1 \mathrm{SS}$ & -1 & 0 & 0 \\
$2 \mathrm{SS}$ & 0 & -1 & 0 \\
$3 \mathrm{SS}$ & 0 & 0 & -1
\end{tabular}

This corresponds to the variant of the game in which Connie comes to the show with her goat and risks losing the pet to Monte in the case of match, when her original guess falls on the cave hiding the goat. A similar $2 \times 2$ matrix is the familiar game of matching pennies, with Monte winning in the event of match (the Quickygnosis article "Matching Pennies" is a good reference).

Going back to the original matrix $C$, we conclude that $V=2 / 3$ is the value of the game, and that the profile

$$
\begin{aligned}
& \boldsymbol{P}^{*}=\left(\frac{1}{3}, 0,0,0, \frac{1}{3}, 0,0,0, \frac{1}{3}, 0,0,0\right), \\
& \boldsymbol{Q}_{1,1,1}^{*}=\left(\frac{1}{3}, 0, \frac{1}{3}, 0, \frac{1}{3}, 0\right)
\end{aligned}
$$

is a solution to the game. The subscript of $\boldsymbol{Q}_{1,1,1}^{*}$ will be explained soon. According to this solution, Monte plays the Monte Crawl strategy: he hides the goat uniformly at random, and he always reveals the Left cave when there is freedom for the second action. Connie selects cave $Y$ uniformly at random and always plays Switch.

A feature of this solution is that the preference of Monte to the Left cave sometimes gives strong confidence to Connie's decision. When Monte reveals the Right cave, he signals that the Left could not be opened, so Connie learns the location of the goat and her Switch action bears no risk.

\section{All Minimax Solutions}

The reader has certainly noticed that strategy $\boldsymbol{Q}_{1,1,1}^{*}$ disagrees with the Little-Endian postulate of Monte's fair-coin-flipping in the event of match, $X=Y$. Monte's behavior at random corresponds to the uniform distribution over all possible choices,

$$
Q_{\frac{1}{2}, \frac{1}{2}, \frac{1}{2}}^{*}=\left(\frac{1}{6}, \frac{1}{6}, \frac{1}{6}, \frac{1}{6}, \frac{1}{6}, \frac{1}{6}\right) .
$$

According to $\boldsymbol{Q}_{\frac{1}{2}, \frac{1}{2}, \frac{1}{2}}^{*}$, Monte hides the goat uniformly at random, and for the second choice between Left and Right if there is freedom a fair coin is flipped. This strategy is also minimax. Furthermore, instead of crawling to the Left cave Monte could be hauled to the Right cave, when there is a choice option.

What are all the minimax strategies of either actor? One way to answer this question is to trace back what was lost in dominated strategy elimination. By column elimination we may delete either of the two pure strategies $X \mathrm{~L}, X \mathrm{R}$ for each $X=1,2,3$. This yields eight minimax solutions $\boldsymbol{Q}_{0,0,0}^{*}$, $\boldsymbol{Q}_{0,0,1}^{*}, \ldots, \boldsymbol{Q}_{1,1,1}^{*}$, where in position $X=1,2,3$ of the index we write 0 if $X \mathrm{~L}$ is never used, and we write 1 if $X \mathrm{R}$ is never used. Each of these solutions is a deterministic behavior either crawling or hauling - depending on the cave by which the match has occurred; these can be considered as mixedaction strategies of Monte, as opposed to his constant-action strategies of always-crawling or always-hauling.

Mixtures of these minimax strategies are again minimax, and each such mixture can be uniquely represented in the form

$$
Q_{\lambda_{1}, \lambda_{2}, \lambda_{3}}^{*}=\left(\frac{\lambda_{1}}{3}, \frac{1-\lambda_{1}}{3}, \frac{\lambda_{2}}{3}, \frac{1-\lambda_{2}}{3}, \frac{\lambda_{3}}{3}, \frac{1-\lambda_{3}}{3}\right)
$$

where $0 \leq \lambda_{X} \leq 1$. The parameter $\lambda_{X}$ has a transparent interpretation: this is the conditional probability that Monte will reveal the Left cave when the goat is hidden behind $X$ and a match $Y=X$ occurs.

The subclass of Monte's strategies with the second action independent of the first given $X=Y$ consists of strategies with equal probabilities $\lambda_{1}=\lambda_{2}=\lambda_{3}$. This mode of Monte's behavior appears as Version Five in Mondee Book [17]. More general strategies $\boldsymbol{Q}_{\lambda_{1}, \lambda_{2}, \lambda_{3}}^{*}$, were also considered in the extended Little-Endian framework [21].

We need to check further whether some minimax strategies for Connie were lost in the course of row elimination. This verification is necessary because the deleted dominated strategies $Y \mathrm{NN}, Y \mathrm{NS}$, and $Y \mathrm{SN}$ are only weakly dominated, meaning that in some situations they perform equally well as the strategies $Y^{\prime}$ SS that dominate them. Examples of games can be given showing that weakly dominated strategies may be minimax (see Course on Games, Section 2.6, Exercise 9).

Recall that a best response is a strategy optimal for an actor knowing which particular strategy the opponent will use. Every minimax strategy $\boldsymbol{P}$ is necessarily a best response to every minimax strategy for Monte, yielding expected payoff 
equal to the value $\boldsymbol{P} \boldsymbol{C} \boldsymbol{Q}_{\lambda_{1}, \lambda_{2}, \lambda_{3}}^{* T}=2 / 3$. Suppose for the time being that minimax strategy $\boldsymbol{P}$ assigns nonzero probability $p>0$ to the pure strategy $2 \mathrm{SN}$, and let $\boldsymbol{P}^{\prime}$ be a strategy obtained from $\boldsymbol{P}$ by removing the $2 \mathrm{SN}$-component but adding weight $p$ to the 1SS-component. Recalling the pattern

\begin{tabular}{c|llllll} 
& $1 \mathrm{~L}$ & $1 \mathrm{R}$ & $2 \mathrm{~L}$ & $2 \mathrm{R}$ & $3 \mathrm{~L}$ & $3 \mathrm{R}$ \\
\hline $1 \mathrm{SS}$ & 0 & 0 & 1 & 1 & 1 & 1 \\
$2 \mathrm{SN}$ & 0 & 0 & 0 & 1 & 1 & 1 \\
$2 \mathrm{NN}$ & 0 & 0 & 1 & 1 & 0 & 0
\end{tabular}

we obtain

$$
\boldsymbol{P}^{\prime} C \boldsymbol{Q}_{\frac{1}{2}, \frac{1}{2}, \frac{1}{2}}^{* T}=P C Q_{\frac{1}{2}, \frac{1}{2}, \frac{1}{2}}^{* T}+\frac{p}{6}>P C Q_{\frac{1}{2}, \frac{1}{2}, \frac{1}{2}}^{* T}
$$

which means that $\boldsymbol{P}^{\prime}$ strictly improves $\boldsymbol{P}$ in combat against the minimax strategy $\boldsymbol{Q}_{1,1,1,2}^{*}$. But this is in contradiction with the assumed minimax property of $\boldsymbol{P}$, and thus 2SN cannot have positive probability in $\boldsymbol{P}$. In the same way it is shown that $2 \mathrm{NN}$ does not enter $\boldsymbol{P}$, and by symmetry among the caves we conclude that none of the dominated strategies enters $\boldsymbol{P}$. Thus nothing was lost by the row elimination.

A crucial property of $Q_{\frac{1}{2}, 2, \frac{1}{2}}^{*}$ we just used is that this strategy gives nonzero probability to each of the six pure strategies for Monte. A mixed strategy $\boldsymbol{Q}$ may be called fully supported if every pure strategy has a positive probability in $\boldsymbol{Q}$. In particular, $Q_{\lambda_{1}, \lambda_{2}, \lambda_{3}}^{*}$, is fully supported if and only if $\lambda_{X}$ is distinct from 0 and 1 for $X=1,2,3$. A best response to a fully supported strategy cannot be weakly dominated, hence minimaxity of some fully supported strategy of Monte precludes minimaxity of every weakly dominated strategy for Connie.

To compare, let us examine the Monte Crawl strategy $\boldsymbol{Q}_{1,1,1}^{*}$, which always reveals the Left cave when there is a match. The pure strategy $1 \mathrm{NS}$ is a best response to $\boldsymbol{Q}_{1,1,1}$, with the winning chance $2 / 3$, as for any other minimax strategy of Connie. If Connie were ensured that Monte will play $\boldsymbol{Q}_{1,1,1}^{*}$ then she may, in principle, choose 1 NS. However, 1 NS versus $\boldsymbol{Q}_{1,1,1}^{*}$ would be an unstable profile, since Monte will then drop Connie's winning chance by swapping to $\boldsymbol{Q}_{\frac{1}{2}, \frac{1}{2}, \frac{1}{2}}^{*}$ (a best response against $1 \mathrm{NS}$ ).

We summarize our analysis of the zero-sum game in the following theorem:

THEOREM The strategy $P^{*}$, which is the uniform mixture of 1SS, 2SS, 3SS, is the unique minimax strategy for Connie. Every strategy $Q_{\lambda_{1}, \lambda_{2}, \lambda_{3}}^{*}$ with $0 \leq \lambda_{X} \leq 1,(X=1,2,3)$ is a minimax strategy for Monte. The value of the game is $V=$ $2 / 3$.

We see that in the setting of zero-sum games any rational behavior of Monte keeps Connie away from employing strategies with Notswitch action. Professor [5] and Magister [7] already knew that $V=2 / 3$ and that throwing symmetric three-sided dice by each of the actors is involved in the solution of the MGG. Our contribution is that the Holy Grail result appears as the first application of the Middle-Endian doctrine of dominance.
The potential of the approach has been further explored by the author $[11,12,13,14]$. In particular, the dominance implies that it is impossible to attribute probabilities to the variables outside of Connie's control in such a way that some strategy utilizing notswitching action will give (strictly) higher winning probability than every alwaysswitching strategy.

\section{Afterword}

The great mathematical minds of the past were not immune to the fallacies of symmetry. The History of the Doctrine of Chances (see [20], p. 48) tells us of Leibniz, who argued that since both $12=6+6$ and $11=5+6$ can be achieved in only one way, with two dice it is as feasible to throw a total 12 points as to throw a total 11. The two-child paradox and the twoenvelopes paradox (consult Quickygnosis) are also of the kind where symmetry is confusing. Most of these "paradoxes" are resolved by adequately setting up the sample spaces and events.

The mystery of switch seems to go beyond just that, and can be only loosely compared with throwing dice, where the uniform distribution is objectively justified by the physical structure of the artifact. Human behavior is a more complex matter, and the assumption of uniform distribution and randomness at all (in the objective, frequentist sense) needs justification, especially when it comes to interaction. Given the model that the actors only care about whether the goat is found or not, the rationality incorporated in the idea of minimax indeed gives a justification for the uniform randomizations both for hiding the goat and the first guess, but it offers no ground for assuming anything about Monte's behavior when he can choose between two empty caves. Moreover, if for some reason Monte exploits a biased random device to hide the goat, Connie still has an optimal alwaysswitching counter-strategy whose performance does not depend on how Monte reveals one of two empty caves.

The world is wrong about the Mondee Gills Game, some people say. Something certainly goes wrong when Laiemen first do not believe that the odds are unequal, then hasten to explain how stupid one is not to see this. What makes the author sceptical about many such explanations is the amazing story about Paul Erdös [16], where in the chapter "Getting the Goat" we read:

Vázsonyi wrote out a "decision tree," not unlike the table of possible outcomes that vos Savant had written out, but this did not convince him. "It was hopeless," Vázsonyi said. "I told this to Erdös and walked away. An hour later be came back to me really irritated. 'You are not telling me why to switch,' he said. 'What is the matter with you?' I said I was sorry, but that I didn't really know why and that only the decision tree analysis convinced me. He got even more upset." Vázsonyi had seen this reaction before [...] but be bardly expected it from the most prolific mathematician of the twentieth century.

This sounds unbelievable, but his why and later pages of the memoirs suggest that the major concern of Erdös was the lack of The Book proof. Using the dominance we do not need any 
probability assumptions in order to discard notswitching, so we hope with this argument we are some steps closer to what Erdös might have wanted to see.

\section{OPEN ACCESS}

This article is distributed under the terms of the Creative Commons Attribution Noncommercial License which permits any noncommercial use, distribution, and reproduction in any medium, provided the original author(s) and source are credited.

\section{REFERENCES}

[1] Dekking, F. M., Kraaikamp, C., Meester, L. E., and Lopuhaä, H. P., A modern introduction in probability and statistics: understanding why and how, Springer, 2005.

[2] Ethier, S. N., The doctrine of chances: probabilistic aspects of gambling, Springer, 2010.

[3] Ferguson, T. S., (1989) Who solved the secretary problem? Statistical Science 4, 282-296.

[4] Ferguson T., (2000) A course on game theory, http://www. math.ucla.edu.

[5] Häggström, O., Streifzüge durch die Wahrscheinlichkeitstheorie, Springer, 2005.

[6] Gill, R., Three door problem...-s, http://arxiv.org/abs/1002.3878, version March 1, 2010.

[7] Gill, R., (2011) The Monty Hall problem is not a probability puzzle (it's a challenge in mathematical modelling), Statistica Neerlandica 65, 58-71.

[8] Gill, R., Monty Hall problem, http://en.citizendium.org/wiki/ Monty_Hall_problem, http://statprob.com/encyclopedia/Monty
HallProblem.html, http://www.math.leidenuniv.nl/ gill/mhp-stat prob.pdf.

[9] Gill, R., The Holy Grail of Monty Hall studies (and other comments) http://www.math.leidenuniv.nl/ gill/.

[10] Gnedin, A., (1994) A solution to the game of googol, Annals of Probability 22, 1588-1595.

[11] Gnedin, A., (2011) The Monty Hall problem in the Game Theory class, arxiv.org.

[12] Gnedin, A., (2011) The Doors, http://arxiv.org/abs/1105.5809.

[13] Gnedin, A., (2011) The Mondee Gills Game: strategic dominance for dummies, preprint.

[14] Gnedin, A., (2011) Dominance in the Monty Hall problem, http://arxiv.org/abs/1106.0833.

[15] Grinstead, C. M., and Snell, J. L., Grinstead and Snell's introduction to probability. http://www.math.dartmouth.edu/prob/ prob/prob.pdf, 2006 (online version of Introduction to Probability, 2nd edition, published by the Amer. Math. Soc.).

[16] Hoffman, P. The man who loved only numbers, Fourth Estate, London, 1998.

[17] Rosenhouse, J. The Monty Hall problem, Oxford University Press, 2009.

[18] Rosenthal, J. S., (2008) Monty Hall, Monty Fall, Monty Crawl. Math Horizons, September issue, 5-7.

[19] Tijms, H., Understanding probability, Cambridge University Press, 2007.

[20] Todhunter, I., A History of the mathematical theory of probability from the time of Pascal to that of Laplace. Chelsea Publishing, 1865.

[21] http://en.wikipedia.org/wiki/Monty_Hall_problem, http://www.scr ibd.com/doc/40800646/Recreational-Math.

[22] http://en.wikipedia.org/wiki/Wikipedia:Arbitration/Requests/Case/ Monty_Hall_problem. 